\title{
Glycosylation of Influenza A Virus Hemagglutinin
}

\author{
$\underline{\text { J. Schwarzer }}^{1}$, M.W. Wolff ${ }^{1}$, E. Rapp ${ }^{1}$, J.K. Schmidt ${ }^{2}$, U. Reichl ${ }^{1,2}$ \\ ${ }^{1}$ Max Planck Institute for Dynamics of Complex Technical System, Bioprocess \\ Engineering, Magdeburg, Germany. \\ ${ }^{2}$ Otto-von-Guericke-University Magdeburg, Bioprocess Engineering, Magdeburg, \\ Germany.
}

The genome of a wide range of viruses, including the influenza A virus, are enclosed in a lipid envelope. These envelopes are generally acquired in the final step of virus assembly. During this step viruses bud from regions of the host cell membranes where virally encoded membrane proteins have accumulated. The influenza A envelope is spiked with two glycoproteins: hemagglutinin (HA) and neuraminidase (NA). HA is the most abundant protein on the virus surface. It plays an important role during virus attachment and membrane fusion. The mature HA forms homotrimers of a monomer which is initially present as a single polypeptide precursor $\left(\mathrm{HA}_{0}\right) . \mathrm{HA}_{0}$ is subsequently cleaved into the subunits: $\mathrm{HA}_{1}$ and $\mathrm{HA}_{2}$. Both subunits are glycosylated and each monomer contains 3 to $9 \mathrm{~N}$-linked glycosylation sequins, depending on the virus strain. The structure of the individual glycans depends mainly on virus subtype and on the host cell.

The biological function of HA-glycans is still not fully clear. However, it has been demonstrated that some of the glycans shield HA from proteases and neutralize antibodies. In addition, they play an important role in the intracellular transport of HA and the viral replication regulation. Structural modifications of the HA-glycans can influence the virus attachment to the host cell and change viral replication dynamics. HA-glycosylation is effected by the glycosylation machinery of the host cell system and can be influenced during vaccine production by choise of the host cell, fermentation-, inactivation- and downstream processing conditions. Therefore, it is important to monitor and control the glycosylation pattern over the period of the production process.

In the present study we determine the HA $N$-glycosylation sites and glycan structure of the influenza A/PR/8/34 (H1N1) virus produced in Madin Darby canine kidney cells (MDCK) cells. Furthermore, the effect of different fermentation conditions on the HA-glycosylation is described. In this model the glycosylation sites of $\mathrm{HA}_{1}$ and $\mathrm{HA}_{2}$ are predicted ( NetNGlyc 1.0 Server ) to be 11, 23, 125 and 283, and 154 and 312, respectively. These sites are confirmed via mass spectrometry.

The glycan structure of the individual sites are characterized via the following procedure. The virus proteins are first separated by SDS-PAGE. $N$-glycans are removed in gel with PNGase F, an enzyme that cleaves Asn-linked oligosaccharides of oligomannosyl, hybrid and complex type. For further analysis by capillary gel electrophoresis (CGE) the $N$-glycans are labelled with 8-Aminopyrene-1,3,6trisulfonic acid trisodium salt (APTS) by reductive amination with $\mathrm{NaBH}_{3} \mathrm{CN}$. CGE is performed on a ABI PRISM 3100-Avant genetic analyser (Applied Biosystems) by laser induced fluorescence. With this method, we are able to fingerprint HA $N$-glycan mixtures from cell culture produced virus with a detection limit of approximately 20 fmol. Additionally HA $N$-glycans are analyzed by mass spectrometry, carried out on a Quadrupol-quadrupol Time-of-flight hybrid mass spectrometer (QStarXL/Applied Biosystems).

For further structural analysis of the HA $N$-glycans is obtained by sequential 
sequencing implementing an reagent array analysis method (RAAM). This involves the progressive digestion of the oligosaccharides with exoglycosidases monitored by CGE and mass spectrometry.

Together, the methods demonstrated here, represent a promising tool to monitor HAglycosylation during the most important steps in upstream and downstream processing of influenza virus for vaccine production. 